\title{
Future of the pharmacogenomics of calc
inhibitors in renal transplant patients
}

\section{"Enzymatic strategies and immunological strategies are the two types of methods which can be used to assess the pharmacodynamics of CNIs. The former directly determines calcineurin activity, while the latter measures immune responsiveness at several levels."}

\section{KEYWORDS: calcineurin inhibitor $\approx$ epigenetics $\approx$ orphan nuclear receptor - pharmacogenomics - renal transplantation}

Since the introduction of calcineurin inhibitors (CNIs), cyclosporine A (CsA) in the early 1980s, and tacrolimus (Tac) in the 1990s, the short-term and long-term survival of kidney grafts have been significantly improved [1]. Nowadays, either CsA or Tac is one of the most commonly used immunosuppressant in renal transplant patients. However, both of them have a narrow therapeutic window and large interindividual variability, resulting in therapeutic drug monitoring (TDM), necessary for adjusting the dose in order to reduce the toxicity and improve the efficacy.

Although TDM is widely recommended in clinical practice and has been conducted for approximately 30 years, this strategy for CNI therapy is controversial according to recent reports [2]. First, the determination of area under the concentration-time curve (AUC) is hard to perform in clinical practice and the evidence for the use of limited sampling strategies is weak [3]. Second, given the drug interactions, diet and polymorphisms potentially attribute to the variability in the pharmacokinetics of CNIs, the start and optimal titration dose for individual patients is difficult to determine based solely on the empirical dose adjustment based on TDM [2]. Third, TDM only provides pharmacokinetic information, and the correlation between pharmacokinetics and pharmacodynamics remains controversial [2]. Thus, more methods should be established to assist TDM and efforts should now be focused on explaining the interindividual variations in the pharmacokinetics and pharmacodynamics of CNIs.

\section{Pharmacogenomics of CNIs in the past decade}

In the past 10 years, understanding of the pharmacogenomics of CNIs in renal transplant patients has been improved. Polymorphisms of genes coding for enzymes and transport proteins involved in the metabolism of CNIs have been thoroughly studied. It is well established that the CYP3A5 A6986G (*3) SNP influences the pharmacokinetics of Tac in renal recipients [4]. Almost all studies have reported that recipients with the $C Y P 3 A 5^{*} 3 /^{*} 3$ genotype (nonexpressers) exhibited higher dose-adjusted Tac exposure $\left(\mathrm{C}_{0} /\right.$ dose, $\mathrm{C}_{2} /$ dose or AUC/dose), and a lower dose requirement compared with the $C Y P 3 A 5^{*} 1 /{ }^{*} 1$ or ${ }^{*} 1 /{ }^{*} 3$ carriers (expressers). Thus the strong association between the $C Y P 3 A 5^{*} 3$ polymorphism and Tac pharmacokinetics could be useful in clinical practice for the determination of dosing strategy. With respect to CsA and the $C Y P 3 A 5^{*} 3$ SNP, the results from clinical studies could not reach a conclusion. A meta-analysis indicated the CYP $3 A 5^{*} 3 \mathrm{SNP}$ was associated with increased CsA dose-adjusted $\mathrm{C}_{0}$ or $\mathrm{C}_{2}$ in renal transplant recipients. In addition, the $C Y P 3 A 5^{*} 3 /{ }^{*} 3$ homozygotes required a lower dose of CsA to reach target levels compared with the $C Y P 3 A 5^{*} 1 /{ }^{*} 1$ or ${ }^{*} 1 /{ }^{*} 3$ genotypes [5]. However, the differences in pharmacokinetics and daily dose appears small between nonexpressers and expressers, limiting the use of the $C Y P 3 A 5^{*} 3 \mathrm{SNP}$ in dose adjustment of CsA [5]. $A B C B 1$, encoding the transport protein P-glycoprotein, which pumps CNIs out of intestinal enterocytes, has had several of its SNPs investigated in renal transplant patients. The influence of these SNPs on the pharmacokinetics of CsA and Tac remains uncertain [4]. As the main enzyme metabolizing CNIs, CYP3A4 also demonstrates interindividual variation in a metabolic capacity [6]. However, functional SNPs are few in the CYP3A4 gene and most studies found no association with pharmacokinetics of CsA, and controversial associations with Tac [4].

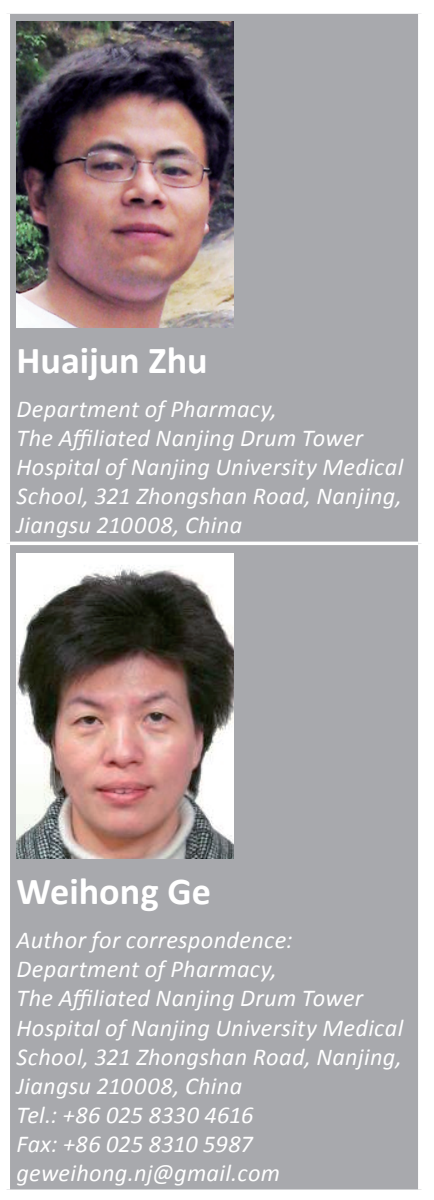

future medicine $_{\text {fisg }}$ 


\section{Pharmacogenomics of CNIs: pharmacological actions}

In the past decade, considerable attention has been paid to the SNPs involved in the CNI metabolism pathway in renal recipients. This may be the result of the accessibility of pharmacokinetic parameters and the popularity of functional experiments on SNPs in metabolic enzymes. As we have described above, the interindividual variation in the pharmacodynamics of CNIs should also be studied on. The biological consequences of CNIs have been defined and both CsA and Tac execute the actions in a similar mechanism. After binding to the intracellular immunophilins, cyclophilin and FK-binding protein 12 (FKBP12), respectively, CsA and Tac inhibit the phosphatase activity of calcineurin, downregulating the dephosphorylation of nuclear factor of activated T cells (NFAT), and reducing the production of the NFAT-regulated genes, such as IL-2 [7].

\section{"Thus, more methods should be established to assist TDM and efforts should now be focused on explaining the interindividual variations in the pharmacokinetics and pharmacodynamics of CNIs."}

However, to date few studies on SNPs involved in the pharmacological actions of CNIs have been conducted. This may be a result of the difficulty of the pharmacodynamic monitoring of CNI therapy. Recently, great efforts have been made to develop such approaches in renal recipients by investigators. Enzymatic strategies and immunological strategies are the two types of methods which can be used to assess the pharmacodynamics of CNIs. The former directly determines calcineurin activity, while the latter measures immune responsiveness at several levels [8]. Although these strategies are still in their infancy and have not been validated in clinical practice, several clinical studies have reported associations of NFATregulated genes with biopsy-proven acute rejection and recurrent infections in renal recipients of CNIs, making the expression of such genes a promising biomarker of pharmacodynamics $[9,10]$. With such progress on pharmacodynamic monitoring, the effect of the SNPs involved in the biological consequences of CNIs on the association between pharmacokinetics and pharmacodynamics could be evaluated in the future. In fact, functional polymorphisms in PPIA (coding for cyclophilin), FKBP1A (coding for FKBP12), PPP3CA/PPP3CB/PPP3R1 (coding for calcineurin), NFATC1/NFATC2/NFAT5 (coding for NFAT) and IL-2 (coding for IL-2) have been explored in other diseases [11]. Nonetheless, further investigations should be conducted to estimate the roles of these SNPs in the pharmacodynamics of CNIs, and more importantly, to ascertain their role in the outcomes of the renal recipients.

\section{Pharmacogenomics of CNIs: orphan nuclear receptors}

Orphan nuclear receptors are a large family of transcription factors regulating tissue gene expression. Evidence in the literature confirmed that many important metabolic enzymes and transporters could be modulated by these receptors, such as the pregnane $\mathrm{X}$ receptor (PXR), constitutive androstane receptor, the glucocorticoid receptor, hepatocyte nuclear factor $4 \alpha$, farnesoid X receptor and the vitamin $\mathrm{D}$ receptor [12]. For example, CYP3A4 mRNA levels have been indicated to be correlated with PXR mRNA levels [13]. Given the interindividual variability of CYP3A4 activity (which could not be explained by the SNPs in CYP3A4), polymorphisms in NR1I2 (coding for PXR) may be the source of the cause and be valuable for further research. Preliminary research has been conducted to examine this and the observation implied that NR1I2 C-25385T significantly influences Tac clearance [14]. The SNPs of not only $P X R$, but also the other nuclear receptors and the combination with the downstream enzymes and transporters may improve the understanding of interindividual variability of $\mathrm{CNI}$ pharmacokinetics, and will this be useful in predicting the optimal dose.

Meanwhile, orphan nuclear receptors could be activated by numerous endogenous and exogenous chemicals found in diet and therapeutic agents. Thus, these receptors play an important role in drug interactions or drug-diet interactions. For instance, phenobarbital could activate PXR, and PXR upregulates CYP3A4, thereby increasing the metabolism of CNIs. By this pathway, PXR modulates the interaction between phenobarbital and CNIs, and the SNPs in the PXR gene may affect this interaction. Accordingly, pharmacogenomics of orphan nuclear receptors will help to predict drug interactions and improve the accuracy of dose prediction if drug combination is present in the renal recipients.

\section{Incorporation of pharmacoepigenetics}

In recent years, with the progress of epigenetics in pharmacology, new specialty pharmacoepigenetics has been developed and raised much attention [15]. Pharmacoepigenetics refers to drug responses 
accounted for by epigenetic changes instead of alterations in the DNA sequence. Epigenetics includes DNA methylation, modification of histones in chromatin and RNA-mediated regulation of gene expression, for example, miRNAs. In contrast to SNPs, epigenetic characteristics could be altered by age, influenced by drugs and can interact with environments. Epigenetic alterations may affect the expression of metabolic enzymes and targets, resulting in the interindividual variability of pharmacokinetics and pharmacodynamics of CNIs.

\section{"Pharmacogenomics of orphan nuclear receptors will help to predict drug interactions and improve the accuracy of dose prediction if drug combination is present in the renal recipients."}

Current evidence has revealed that the expression of CYP3A4 and CYP3A5 could be affected by a DNA methyltransferase inhibitor and miRNA$27 \mathrm{~b}[16,17]$. Besides the direct action on enzymes, miRNAs also regulate the expression of nuclear receptors, such as PXR [18]. With regard to the pharmacodynamics, few data has been reported. Experiments have suggested that miRNA-145 could bind to the 3'-UTR of PPP3CA and regulate its expression [19]. In silico analysis has also revealed the presence of several binding sites of miRNAs in the 3'-UTRs of PPP3R1 and NFAT5 [20]. In view of the advancement of pharmacoepigenetics, epigenetic characteristics could be determined in renal recipients and the association with responses of CNIs could be evaluated. Results of such studies will offer another level of explanation for interindividual variations of CNIs and optimal dosing strategy will be improved. Notably, epigenetics has tissue specificity and the determination of epigenetic characteristics appears to be a barrier for pharmacokinetic studies owing to a difficulty obtaining the hepatic tissue of recipients. However, pharmacoepigenetic studies on pharmacodynamics of CNIs are feasible since the biological effects of CNIs take place in lymphocytes.

\section{Summary \& future perspective}

In summary, although pharmacogenomic studies have validated some associations of SNPs with the pharmacokinetics of CNIs, further valuable work is still needed to explore the interindividual variations of drug response. In the future, more attention should be paid to the pharmacodynamics, to widen the research, and to the polymorphisms in genes coding orphan nuclear receptors, to deepen the research. Meanwhile, the incorporation of pharmacoepigenetics into pharmacogenomic studies will help to disclose the factors contributing to the interindividual variations of CNIs. Through these research results, more reliable and accurate optimal dosing strategies will be expected and applied in the clinical practice.

\section{Financial \& competing interests disclosure}

This work was supported by the grant from the Medical Science and Technology Development Foundation of Nanjing Department of Health (grant YKK10060). The authors have no other relevant affiliations or financial involvement with any organization or entity with a financial interest in or financial conflict with the subject matter or materials discussed in the manuscript apart from those disclosed.

No writing assistance was utilized in the production of this manuscript.

\section{Bibliography}

1 Hariharan S, Johnson CP, Bresnahan BA, Taranto SE, McIntosh MJ, Stablein D. Improved graft survival after renal transplantation in the United States, 1988 to 1996. N. Engl. J. Med. 342(9), 605-612 (2000).

2 Sommerer C, Giese T, Meuer S, Zeier M. Pharmacodynamic monitoring of calcineurin inhibitor therapy: is there a clinical benefit? Nephrol. Dial. Transplant. 24(1), 21-27 (2009).

3 Van Rossum HH, Press RR, den Hartigh J, de Fijter JW. Point: a call for advanced pharmacokinetic and pharmacodynamic monitoring to guide calcineurin inhibitor dosing in renal transplant recipients. Clin. Chem. 56(5), 732-735 (2010).
4 Staatz CE, Goodman LK, Tett SE. Effect of $C Y P 3 A$ and $A B C B 1$ single nucleotide polymorphisms on the pharmacokinetics and pharmacodynamics of calcineurin inhibitors: part I. Clin. Pharmacokinet. 49(3), 141-175 (2010).

5 Tang HL, Ma LL, Xie HG, Zhang T, Hu YF. Effects of the $C Y P 3 A 5^{*} 3$ variant on cyclosporine exposure and acute rejection rate in renal transplant patients: a meta-analysis. Pharmacogenet. Genomics. 20(9), 525-531 (2010).

6 Hirota T, Ieiri I, Takane $\mathrm{H}$ et al. Allelic expression imbalance of the human CYP3A4 gene and individual phenotypic status. Hum. Mol. Genet. 13(23), 2959-2969 (2004).

7 Vicari-Christensen M, Repper S, Basile S, Young D. Tacrolimus: review of pharmacokinetics, pharmacodynamics, and pharmacogenetics to facilitate practitioners' understanding and offer strategies for educating patients and promoting adherence. Prog. Transplant. 19(3), 277-284 (2009).

8 Van Rossum HH, de Fijter JW, van Pelt J. Pharmacodynamic monitoring of calcineurin inhibition therapy: principles, performance, and perspectives. Ther. Drug Monit. 32(1), 3-10 (2010).

9 Sommerer C, Zeier M, Meuer S, Giese T. Individualized monitoring of nuclear factor of activated $\mathrm{T}$ cells-regulated gene expression in FK506-treated kidney transplant recipients. Transplantation 89(11), 1417-1423 (2010).

10 Sommerer C, Giese T, Schmidt J, Meuer S, Zeier M. Ciclosporin A tapering monitored by NFAT-regulated gene expression: a new 
concept of individual immunosuppression. Transplantation 85(1), 15-21 (2008).

11 Press RR, de Fijter JW, Guchelaar HJ. Individualizing calcineurin inhibitor therapy in renal transplantation-current limitations and perspectives. Curr. Pharm. Des. 16(2), 176-186 (2010).

12 Urquhart BL, Tirona RG, Kim RB. Nuclear receptors and the regulation of drug-metabolizing enzymes and drug transporters: implications for interindividual variability in response to drugs. J. Clin. Pharmacol. 47(5), 566-578 (2007).

13 Westlind-Johnsson A, Malmebo S, Johansson A. Comparative analysis of CYP3A expression in human liver suggests only a minor role for CYP3A5 in drug metabolism. Drug Metab. Dispos. 31(6), 755-761 (2003).
14 Benkali K, Prémaud A, Picard N et al. Tacrolimus population pharmacokineticpharmacogenetic analysis and Bayesian estimation in renal transplant recipients. Clin. Pharmacokinet. 48(12), 805-816 (2009).

15 Peedicayil J. Pharmacoepigenetics and pharmacoepigenomics. Pharmacogenomics 9(12), 1785-1786 (2008).

16 Dannenberg LO, Edenberg HJ. Epigenetics of gene expression in human hepatoma cells: expression profiling the response to inhibition of DNA methylation and histone deacetylation. BMC Genomics 7, 181 (2006).

17 Pan YZ, Gao W, Yu AM. MicroRNAs regulate CYP3A4 expression via direct and indirect targeting. Drug Metab. Dispos. 37(10), 2112-2117 (2009).
18 Takagi S, Nakajima M, Mohri T, Yokoi T. Post-transcriptional regulation of human pregnane $\mathrm{X}$ receptor by micro-RNA affects the expression of cytochrome P450 3A4. J. Biol. Chem. 283(15), 9674-9680 (2008).

19 Ostenfeld MS, Bramsen JB, Lamy P et al. miR-145 induces caspase-dependent and -independent cell death in urothelial cancer cell lines with targeting of an expression signature present in Ta bladder tumors. Oncogene 29(7), 1073-1084 (2010).

20 Asirvatham AJ, Gregorie CJ, Hu Z, Magner WJ, Tomasi TB. MicroRNA targets in immune genes and the Dicer/Argonaute and ARE machinery components. Mol. Immunol. 45(7), 1995-2006 (2008). 